\section{Evaluation of Combined Effects of Insomnia and Stress on Sleep Quality and Sleep Duration}

\section{Abstract}

Objectives: Insomnia and Stress impacts both neural development of central nervous structures, and they are involved in many clinical outcomes like sleep disorders, depression, cognitive impairment and other sleep disorders. Few authors investigated accurately relationship between implications of these two troubles above, and occurrence or complications of sleep quality. It has been showed that, the quality and the duration of sleep depended strongly on the lifestyle and the personal environment. Knowing that, insomnia and stress are a permanent part of our daily life, a complete exploration of their combined effects on our sleep quality and sleep duration may move forward the general approach of therapist about sleep impairment. Indeed, a complete evaluation should be made continuously on a brain to record all the waves fluctuations related to sleep, but an early detection of factors influencing these changes in cerebral wave may be equally interesting. To our knowledge, none made a clear statement on the combined effects of insomnia and stress on the Sleep Impairment events. The aim of this study is to explore how insomnia and stress together affects sleep quality and sleep duration (SD), in the general population is associated in simultaneous with sleep components, psychological stress, depression, anxiety, well-being, addiction and global health of participants; and if it is also influenced by the sociodemographic profile of each subject.

Methods: The present study was led by the questionnaire Mental Health Profile of Etindele (MHPE) incorporating McNair test, and incorporated sub-score for sleep components, psychological stress, depression, anxiety, well-being, addiction, family history of the participant and his family. All this clinical and environmental measure were associated with sociodemographic profile of each participant, to address our question Our results showed that the stress level and sleep duration are comparable per gender and family history.

Results: Sleep quality was correlated strongly with decrease of clinical parameters, and the level of stress. the results showed that lack of sleep combined with a low global score to MHPE are strongly correlated with sleep impairment, while score of memory and attention decreased with insomnia. Insomnia is strongly correlated with physical activity.

Conclusion: Insomnia and stress together speed up decline of memory and sleep impairments.

Keywords: Sleep duration; Sleep quality; Insomnia; Stress level; MHPE; Physical activity; Sleep impairment

\section{Faustin Armel Etindele Sosso ${ }^{1,2 *}$ Okito Nakamura ${ }^{3}$ and Mitsu Nakamura ${ }^{4}$}

1 Research Center in Neuropsychology and Cognition, Quebec, Canada

2 Department of Biological Sciences, University of Montreal, Quebec, Canada

3 Global Research Department, Ritsumeikan University Kyoto, Japan

4 The graduated University of Advanced Studies, Miura Japan

*Corresponding author:

Faustin Armel Etindele Sosso, PhD.

faustin.armel.etindele.sosso@ umontreal.ca

Research Center in Neuropsychology and Cognition, Quebec, 90, Avenue Vincent d'Indy, H2V2S9, Canada.

Tel: +1 $5143436111 \# 3187$

Citation: Etindele Sosso FA, Nakamura O, Nakamura M (2017) Evaluation of Combined Effects of Insomnia and Stress on Sleep Quality and Sleep Duration. J Neurol Neurosci. Vol. 8 No. 4: 203. 


\section{Introduction}

Sleep Impairments (SI) may appear anytime at any step of human development; toughness to elderly [1]. During the entire life, cerebral structures involved in sleep are particularly influenced by lifestyle and environmental stressing factors which modulate; the neuronal responses of our brain [2]. This interaction hardly weighs on the maintain of a regular good sleep, with an appropriate duration [3]. Indeed, most of the mental disorders are caused by a dysfunction in one or many physiological modulation [4]. Because of these dysfunctions, SI are generated and their clinical outcome may be moderate or chronic [5]. An healthy lifestyle with regular physical activity may ensures a healthy brain, and an excellent shield against both peripheral and central nervous disorders, as well as and cognitive decline [6].

Recent researches reported that brain structures involved in sleep mechanism are modulated by the balance between inhibitory neuronal system and excitation neuronal circuitry [7]. Insomnia and Stress impacts both this neural development of central nervous structures, and they are involved in many clinical outcomes like sleep disorders [8], depression [9] and cognitive impairment or mood disorders [9]. Few authors investigated accurately relationship between implications of these two troubles above, and occurrence or complications of sleep disorders [10-12]. It has been showed that, the quality and the duration of sleep depended strongly of the lifestyle and the personal environment $[9,13-16]$. Knowing that, insomnia and stress are a permanent part of our daily life, a complete exploration of their combined effects on our sleep quality [1] and sleep duration (SD) may move forward the general approach of therapist about sleep impairment. To our knowledge, none made a clear statement on the combined effects of insomnia and stress on the SI events. The aim of this study is to explore how insomnia and stress together affects SQ and SD.

\section{Materials and Methods}

\section{Ethics committee}

The present research was approved beforehand, by the committee of ethic and research of the faculty of arts and science of the University of Montreal, in Quebec, Canada. All our volunteering participants signed a consenting form, before the study.

\section{Sample, Parameters and Data Analysis}

Socio-demographic and clinical information about age, medication in progress for insomniac trouble, gender, education, medical history of the participant's family, memory deficiency and cognitive complaints were collected with the Mental Health Profile of Etindele questionnaire (MHPE). This questionnaire was employed in previous published studies, and it is precise and sensitive for the detection of the parameter measured. Current and past histories of medications were classified as medications of musculoskeletal, neurological, respiratory or cardiovascular disease. McNair scale was calculated using the shorter version of 15 items. Subjects aged more than 50 years old, enabled to complete the program and speaking other native language than
French and English were removed from analysis $(n=6)$. SD and SQ were evaluated with seven items; sleep duration self reported, use of sleeping pills, history of medications, medication length, starting of sleeping disorders, sleep quality self reported ranged from 1 "very bad" to 5 "very well" and the difficulty of falling asleep from 1" No" to 4 "too much". 100 respondents were assessed in subjective SD and SQ using our MHPE questionnaire. The self-report questions for SD and SQ included 20 items (scored from 0 "no" to 3 "very difficult"). Insomnia was then identified over the global score ranging from 0 (no) to 60 (chronic). Scores between 25 to 45 points were considered indicative of moderate insomnia, and from 46 to 60 was categorised like chronic event of insomnia. Normality was tested with the Bartlett's test. The analysis of the MHPE response are like previous published work [15] (Table 1).

\section{Results}

100 subjects were used in the study. The response to the questionnaire was maximum. $80 \%$ of the sample was aged between 18 and 30 years, an expected proportion. Women represented $50 \%$ of the sample. Majority of the respondents were graduate students (60\%).

Looking deeply the family history's disease, $23 \%(n=23)$ suffered from cardiovascular disease and $12 \%(n=12)$ suffered from neurologic disease. $64 \%(n=64)$ of them, have family members with insomnias and $35 \%(n=35)$ suffered from Alzheimer. $8 \%$ $(n=8)$ of respondents were treated from cardiovascular disease, $18 \%(n=18)$ suffered from musculoskeletal disease, $15 \%(n=15)$ used medication for neurologic disease and $3 \%(n=3)$ has an abdominal impairment. All clinical parameters were associated with insomnia ( $p<0.00041$, Kruskal Wallis test) except for the usage of drugs or memory impairment ( $p=0.074, U$ MannWhitney's test). The analysis of depression and anxiety showed that $69 \%(n=69)$ of the participants has a depression but $16 \%$ $(n=16)$ has an anxiety. The average well-being score was $19 \pm 0.8$ with a good correlation with McNair score $(p<0.0001$, spearman rank). The mean dependency score was $17.45 \pm 0.58$ (SD), based on Spearman rank it's associated with McNair score $(p=0.00023)$. However, there was no correlation between well-being score, stress score and McNair $(p=0.61, p=0.0057$ respectively with Spearman rank test).

Results showed a good correlation between stress level and sleep components except for the beginning of sleep disturbances $(p=0.439, U$ Mann Whitney test). The logistic regression was executed on uncorrelated variables to identify the best predictors for SD and SQ. Multicollinearity was detected between the parameters: sleeping pills, medication, and beginning of sleep disturbances. The following variables were included: depression, anxiety, duration of medication, physical activity and insomnia. The analysis revealed that $10 \%$ of the variation in SI was justified by sleep duration, medication length, stress and insomnia. The model was significant $(p<0.005)$.

The variable SQ was the least significant predictor in this model (Wald statistic $=33.25$, $p$-value $<0.0001$ ) and SD the most significant $($ Wald statistic $=102.91, p$-value $<0.0001)$. Insomnia 
Table 1 Relationships between MHPE score, demographic and clinical characteristics.

\begin{tabular}{|c|c|c|}
\hline Participants profile & $\begin{array}{l}\text { Mean } \pm \text { SD } \\
\text { Or } n(\%)\end{array}$ & $\begin{array}{l}\text { MHPE score } \\
\text { Mean } \pm \text { SD }\end{array}$ \\
\hline \multicolumn{3}{|c|}{ Age } \\
\hline $18-24$ & $60(60 \%)$ & $24.3 \pm 0.21$ \\
\hline $24-30$ & $20(20 \%)$ & $38.45 \pm 0.39$ \\
\hline $30-36$ & $20(20 \%)$ & $33 \pm 1.23$ \\
\hline \multicolumn{3}{|c|}{ Gender } \\
\hline Male & $50(50 \%)$ & $42.6 \pm 0.309$ \\
\hline Female & $50(50 \%)$ & $44.75 \pm 0.219$ \\
\hline \multicolumn{3}{|c|}{ Level of education } \\
\hline First cycle & $11(11 \%)$ & $34.18 \pm 0.23$ \\
\hline Secondary cycle & $60(60 \%)$ & $33.60 \pm 0.238$ \\
\hline Third cycle & $20(20 \%)$ & $45.30 \pm 0.458$ \\
\hline $\begin{array}{l}\text { Else (certificat. AEC. DEP. } \\
\text { microprogramme) }\end{array}$ & $9(9 \%)$ & $14.84 \pm 0.68$ \\
\hline \multicolumn{3}{|c|}{ Medication history (Cognitive or memory impairment drugs) } \\
\hline Yes & $64(64 \%)$ & $43 \pm 0.504$ \\
\hline No & $36(36 \%)$ & $14.30 \pm 0.191$ \\
\hline \multicolumn{3}{|c|}{$\begin{array}{l}\text { Family history of neurological, musculoskeletal, respiratory or } \\
\text { cardiovascular disease (1-12 months) }\end{array}$} \\
\hline Cardiovascular disease & $23(23 \%)$ & $12.88 \pm 0.225$ \\
\hline Musculoskeletal disease & $18(18 \%)$ & $22 \pm 0.756$ \\
\hline Neurologic disease & $12(12 \%)$ & $43 \pm 0.494$ \\
\hline Respiratory disease & $11(11 \%)$ & $20 \pm 0.87$ \\
\hline Other & $19(19 \%)$ & $25.83 \pm 0.493$ \\
\hline None & $11(11 \%)$ & $14.09 \pm 0.412$ \\
\hline \multicolumn{3}{|c|}{ Family's history for cognitive or memory impairments (1-12 months) } \\
\hline Memory deficiency & $36(36 \%)$ & $34.33 \pm 0.491$ \\
\hline Attention deficit disorders & $12(12 \%)$ & $23.75 \pm 0.221$ \\
\hline Alzheimer & $8(8 \%)$ & $69 \pm 0.233$ \\
\hline Cognitive impairments & $14(14 \%)$ & $44.86 \pm 0.258$ \\
\hline Other & $3(3 \%)$ & $27 \pm 1.35$ \\
\hline None & $27(27 \%)$ & $13 \pm 0.226$ \\
\hline Medication. current & & \\
\hline \multicolumn{3}{|c|}{$\begin{array}{l}\text { Medication of neurological, musculoskeletal, respiratory or } \\
\text { cardiovascular disease (1-12 months) }\end{array}$} \\
\hline Cardiovascular disease & $32(32 \%)$ & 16 \\
\hline Musculoskeletal disease & $32(32 \%)$ & 21 \\
\hline Neurologic disease & $8(8 \%)$ & $32.5 \pm 0.315$ \\
\hline Respiratory disease & $16(16 \%)$ & $14 \pm 0.22$ \\
\hline Other & $12(12 \%)$ & $12 \pm 0.825$ \\
\hline \multicolumn{3}{|c|}{ Depression } \\
\hline Normal & $64(64 \%)$ & $14.62 \pm 0.19$ \\
\hline Moderate & $16(16 \%)$ & $15 \pm 0.8$ \\
\hline Mild & $20(20 \%)$ & $40 \pm 0.8$ \\
\hline Severe & $0(0 \%)$ & $\mathrm{n} / \mathrm{a}$ \\
\hline \multicolumn{3}{|c|}{ Anxiety } \\
\hline Normal & $48(48 \%)$ & $15.5 \pm 0.35$ \\
\hline Moderate & $28(28 \%)$ & $24.86 \pm 0.26$ \\
\hline Mild & $18(18 \%)$ & $32.9 \pm 0.31$ \\
\hline Severe & $12(12 \%)$ & $41 \pm 0.8$ \\
\hline
\end{tabular}

(Wald statistic $=77.8, p$-value $<0.0001)$, duration of medication between one month and one year ( $p$-value $<0.0001$ vs 1 year, $p=0.075$ ), no difficulty of falling asleep (Wald statistic $=77.7$, $p$-value $=0.001$ ) or have a difficulty to fall asleep ( $p$-value $<0.0023$ vs a little difficulty to fall asleep, $p$-value $=0.436)$, mild subjective sleep self-report satisfaction ( $p$-value $<0.0001)$ were correlated with cognitive decline (Table 2).

\section{Discussion}

Simultaneous side effect of stress and the sleep components SD and SQ, were easily visible in our findings. This combination was not followed continuously, but current results showed an increase of cognitive decline, as well as SI in our statistic population. One goal of the current research is to provide a new approach in the detection of SI, by a rapid and easy evaluation of SD and SQ. Several authors reported crucial role of environmental risk factors specifically stress, which could impair the balance of mental health [9,14-16]. At our knowledge, there are fewer evidence of correlation between simultaneous effects of the clinical parameters computed above, on the apparition of insomnia and impairment of sleep components [17]. It has been demonstrated that, physical exercise like aerobic and dance; are good shield against stress and cognitive decline mechanism [18,19], while an appropriate SQ and SD certainly helps in consolidation of memory and integration of additional information in the synaptic circuitry [20]. Previous published researches showed the impact of what is known now as complex combination, on the brain disorders [15]. The present findings are in line with them, also with other studies showing an association between many components of this complex combination with salacious decline of cognitive functions as well as sleep components. Issue is appropriate literature on the present association needs more investigation, from basic mechanism until clinical testing. Our findings suggest existence of a modulating balance of stress and insomnia, on

Table 2 Relationships between sleep components and stress score.

\begin{tabular}{|c|c|c|c|c|}
\hline & $\boldsymbol{\beta}$ & SE & OR ( $95 \%$ C.I.) & p-value \\
\hline \multicolumn{5}{|c|}{ Sleep duration } \\
\hline $4 \mathrm{~h}$ & -1.439 & 0.395 & $0.237(0.109 / 0.514)$ & 0.000 \\
\hline $5 \mathrm{~h}$ & -2.667 & 0.352 & $0.069(0.035 / 0.138)$ & 0.000 \\
\hline $6 \mathrm{~h}$ & -0.803 & 0.243 & $0.448(0.278 / 0.720)$ & 0.001 \\
\hline $7 \mathrm{~h}$ & -1.017 & 0.200 & $0.362(0.244 / 0.535)$ & 0.000 \\
\hline \multicolumn{5}{|c|}{ Duration of medication } \\
\hline None & 0.440 & 0.212 & $1.552(1.024 / 2.353)$ & 0.038 \\
\hline Less than month & 3.709 & 0.458 & $40.807(16.630 / 100.134)$ & 0.000 \\
\hline $\begin{array}{c}1 \text { month - } 6 \\
\text { months }\end{array}$ & 2.359 & 0.316 & $10.578(5.693 / 19.653)$ & 0.000 \\
\hline 6 months - 1 year & 20.759 & 7105.18 & 11.33.09.62.20.169 & 0.998 \\
\hline \multicolumn{5}{|c|}{ Sleep quality } \\
\hline Very bad & -20.052 & 7105.18 & 0 & 0.998 \\
\hline Bad & 0.382 & 0.430 & 0 & 0.374 \\
\hline Mild & 0.955 & 0.276 & $2.598(1.512 / 4.462)$ & 0.001 \\
\hline Well & -0.085 & 0.244 & 0 & 0.728 \\
\hline Very well & & & & \\
\hline \multicolumn{5}{|c|}{ Difficulty falling a sleep } \\
\hline None & 1.080 & 0.370 & $2.945(1.427 / 6.077)$ & 0.003 \\
\hline little & 0.153 & 0.384 & 0 & 0.690 \\
\hline Difficult & -1.731 & 0.395 & $0.177(0.082 / 0.384)$ & 0.000 \\
\hline Very difficult & - & - & - & - \\
\hline
\end{tabular}


sleep components in general; and on SD and SQ. People with less than 6 hours of study and a moderate anxiety, has a low global score on MHPE and weak score in all his sub sections. This result is also the same, in items related to insomnia. This longitudinal research confirms the hypothesis that during learning process; neuronal consolidation is more stabilized by an appropriate SD [21]. It is also possible that, insomnia is a consequence of a silent damage of the brain structure. The findings suggested it is possible to anticipate sleep disorders, even with people without a medical diagnostic, with the MHPE. Following our observations, a regular evaluation of insomnia, SD, SQ and associated clinical outcomes likes depression; while controlling risk factors and lifestyle; will definitively improves promotion and prevention of sleep disorders (Table 3).

Table 3 Logistic regression analysis of the association between subjective Insomnia and sleep components.

\begin{tabular}{|c|c|c|}
\hline Sleep components & Parameters & $\begin{array}{l}\text { Stress score } \\
\text { Mean } \pm \text { SD }\end{array}$ \\
\hline \multirow{6}{*}{ Sleep duration } & $4 \mathrm{~h}$ & $46 \pm 0.63$ \\
\hline & $5 \mathrm{~h}$ & $35.75 \pm 0.58$ \\
\hline & $6 \mathrm{~h}$ & $25.42 \pm 0.42$ \\
\hline & $7 \mathrm{~h}$ & $17.6 \pm 2.3$ \\
\hline & $8 \mathrm{~h}$ & $13.06 \pm 0.26$ \\
\hline & More than $8 \mathrm{~h}$ & $16.73 \pm 0.85$ \\
\hline \multirow{2}{*}{ Sleeping pills } & Yes & 23 \\
\hline & No & $14.02 \pm 0.185$ \\
\hline \multirow{7}{*}{ Medication } & None & $13.54 \pm 0.23$ \\
\hline & antibiotics & $17.56 \pm 0.76$ \\
\hline & antidepressants & $43.06 \pm 0.28$ \\
\hline & $\begin{array}{c}\text { vitamins or energetic } \\
\text { drinks }\end{array}$ & $12.29 \pm 0.415$ \\
\hline & $\begin{array}{l}\text { Acupuncture or } \\
\text { hypnosis }\end{array}$ & $26.06 \pm 0.2$ \\
\hline & $\begin{array}{l}\text { Anxiolytics or sleeping } \\
\text { pills }\end{array}$ & $36 \pm 1.88$ \\
\hline & anti-inflammatory & $18.50 \pm 0.183$ \\
\hline \multirow{5}{*}{ Duration of medication } & None & $13.52 \pm 0.23$ \\
\hline & Less than one month & $37.5 \pm 0.59$ \\
\hline & 1 month - 6 months & $14.25 \pm 0.54$ \\
\hline & 6 months - 1 year & $26 \pm 1.04$ \\
\hline & More than one year & $43.29 \pm 0.34$ \\
\hline \multirow{5}{*}{ Sleep quality } & Very bad & $24 \pm 0.69$ \\
\hline & Bad & $15 \pm 0.71$ \\
\hline & Mild & $14.75 \pm 0.29$ \\
\hline & Well & $9.62 \pm 0.28$ \\
\hline & Very well & $5.4 \pm 0.34$ \\
\hline \multirow{4}{*}{ Difficulty falling a sleep } & None & $7 \pm 0.26$ \\
\hline & little & $12 \pm 0.28$ \\
\hline & Difficult & $12.60 \pm 0.48$ \\
\hline & Very difficult & $22.33 \pm 0.81$ \\
\hline
\end{tabular}

Considering the present results, it was not possible to follow regularly the participants, and record continuously evolution of insomnia, during the following weeks. In an upcoming study, which is currently in progress, three interviews are plan with participants (equally spaced of one month). The MHPE will be using each interview to estimate changes of the clinical parameters and all sleep components. Expecting our sample number stay the same, more variation and precise estimation will emerge.

\section{Conclusion}

A healthy brain is dependent of appropriate SD and SQ. Obviously, physiological behavior and environmental stressors are not the same for everybody. Many investigations should be done to clarify the exacts effects and how to handle them. 


\section{References}

1 Leite AH, Almeida TL, Mesquita AR (2009) The impact of age on emotional and cognitive behaviours triggered by experimental neuropathy in rats. Pain 144: 57-65.

2 Burke TM, Scheer FA, Ronda JM, Czeisler CA, Wright KP, et al. (2015) Sleep homeostatic and circadian influences on higher-order cognitive functions. J Sleep Res 24: 364-371.

3 Daulatzai MA (2015) Evidence of neurodegeneration in obstructive sleep apnea: relationship between obstructive sleep apnea and cognitive dysfunction in the elderly. J Neurosci Res 93: 1778-1794.

4 Edwards KM, Kamat R, Tomfohr LM, Ancoli-Israel S, Dimsdale JE (2014) Obstructive sleep apnea and neurocognitive performance: the role of cortisol. Sleep Med 15: 27-32.

5 Elcombe EL, Lagopoulos J, Duffy SL (2015) Hippocampal volume in older adults at risk of cognitive decline: the role of sleep, vascular risk, and depression. J Alzheimers Dis 44: 1279-1290.

6 Foster PP (2015) Role of physical and mental training in brain network configuration. Front Aging Neurosci 7: 117.

7 Ferini SL, Galbiati A, Marelli S (2013) Sleep microstructure and memory function. Front Neurol 4: 159.

8 Fernandez MJ (2017) The insomnia with short sleep duration phenotype: an update on it's importance for health and prevention. Curr Opin Psychiatry 30: 56-63.

9 Etindele Sosso FA, Neurocognitive game between risk factors, sleep and suicidal behaviour (Sleep Science 2017)

10 Chahine LM, Amara AW, Videnovic A (2016) A systematic review of the literature on disorders of sleep and wakefulness in Parkinson's disease from 2005 to 2015. Sleep Med Rev.
11 Cunnington D, Junge $\mathrm{M}$ (2016) Chronic insomnia: Diagnosis and nonpharmacological management. BMJ 355: i5819.

12 Maski K, Owens JA (2016) Insomnia, parasomnias, and narcolepsy in children: clinical features, diagnosis, and management. Lancet Neurol 15: 1170-1181.

13 Goerke M, Muller NG, Cohrs S (2015) Sleep-dependent memory consolidation and its implications for psychiatry. J Neural Transm (Vienna).

14 Sosso FAE (2017) Sleep disorders and insomnia: effects on a young population. ECPP 2: 26-32.

15 Etindele Sosso FA, Raouafi S (2016) Brain disorders:correlation between cognitive impairment and complex combination. Ment Health Fam Med 12: 215-222.

16 Etindele Sosso F, Raouafi S (2016) Appropriate sleep duration and physical activity modulate cognitive improvement. J Sleep Disor: Treat Care 5: 4.

17 Jan JE, Reiter RJ, Bax MC, Ribary U, Freeman RD, et al. (2010) Longterm sleep disturbances in children: a cause of neuronal loss. Eur $\mathrm{J}$ Paediatr Neurol 14: 380-390.

18 Yu F, Xu B, Song C, Ji L, Zhang X (2013) Treadmill exercise slows cognitive deficits in aging rats by antioxidation and inhibition of amyloid production. Neuroreport 24: 342-347.

19 Buchman AS, Boyle PA, Yu L, Shah RC, Wilson RS, et al. (2012) Total daily physical activity and the risk of $A D$ and cognitive decline in older adults. Neurology 78: 1323-1329.

20 Cellini N (2016) Memory consolidation in sleep disorders. Sleep Med Rev.

21 Kay SM, Attarian H (2016) Advances in the management of chronic insomnia. BMJ 354: i2123. 